\title{
Effects of MHD and Porosity on Jeffrey Fluid Flow with Wall Transpiration
}

\author{
Sana Bajwa, ${ }^{1}$ Saif Ullah $\left(\mathbb{D},{ }^{1}\right.$ Amnah S. Al-Johani, ${ }^{2}$ Ilyas Khan $\left(\mathbb{D},{ }^{3}\right.$ \\ and Mulugeta Andualem $\mathbb{D}^{4}$ \\ ${ }^{1}$ Department of Mathematics, Government College University Lahore, Lahore 54000, Pakistan \\ ${ }^{2}$ Mathematics Department, Faculty of Science, University of Tabuk, Tabuk, Saudi Arabia \\ ${ }^{3}$ Department of Mathematics, College of Science Al-Zulfi, Majmaah University, Al-Majmaah 11952, Saudi Arabia \\ ${ }^{4}$ Department of Mathematics, Bonga University, Bonga, Ethiopia
}

Correspondence should be addressed to Ilyas Khan; i.said@mu.edu.sa and Mulugeta Andualem; mulugetaandualem4@gmail.com

Received 1 September 2021; Revised 19 January 2022; Accepted 1 February 2022; Published 25 February 2022

Academic Editor: Zhengbiao Peng

Copyright (C) 2022 Sana Bajwa et al. This is an open access article distributed under the Creative Commons Attribution License, which permits unrestricted use, distribution, and reproduction in any medium, provided the original work is properly cited.

In this research work, the velocity of Jeffrey fluid flow which is unsteady in nature, over an infinite horizontal porous plate, is considered, and also, the influence of MHD and porosity on the velocity of the fluid is investigated. The major objective of this research paper is to achieve the analytical solution for the incompressible transient flow of MHD (magneto hydrodynamic) Jeffrey fluid above an accelerating porous plate. The fluid flows above the plate at $y>0$, and the plate is infinite in the $x$-direction $(y=0)$ and gives an oscillatory motion. For the occurrence of both injection and suction phenomena, porous plate is used. The governing equation of the model becomes dimensionless by using the appropriate set of nondimensional variables. With the help of Laplace transformation and perturbation methods, these nondimensional differential equations of Jeffrey fluid are solved. The effects of different parameters on velocity are studied. These parameters include $\omega$ (oscillating frequency), $\lambda 1$ (ratio of relaxation and retardation time), $\beta$ (Jeffrey fluid parameter), $\gamma$ (wall transpiration parameter), $t$ (time), $k$ (porosity parameter), and Ha (magnetic parameter). We found that velocity declines as we increase the values of ratio of relaxation and retardation times, Jeffrey fluid parameter, wall transpiration parameter, and magnetic parameter. The effect of time, oscillation frequency, and porosity on velocity profile is opposite as that of other parameters. We take $\beta<1$, as $\beta$ represents perturbed parameter. Graphs are plotted for various values of these parameters with the help of Mathcad software and talk over these graphs in detail.

\section{Introduction}

It is scrutinized since the past limited eras that several scholars are far fascinated in non-Newtonian fluids. The clarification behind such curiosity in non-Newtonian fluid is because of its wide-ranging scope in many fields of life. NonNewtonian fluids have numerous uses and applications in many fields, for example, chemical industries, biological sciences, geophysics, and petroleum. As we are aware all non-Newtonian fluids have attained the properties of elasticity along with viscosity. The countless models of nonNewtonian fluids are present in our daily life activities, such as oils, ketchup, honey, paints, and toothpaste, and asphalt, and liquid polymers are characterized by some noteworthy phenomena. These fluids are a number of thought-provoking applications and are moreover used in our daily life. The nonlinear relationship between shear stress and shear rate in such types of fluids is verified by many researchers that is not only significant from an academic point of view but also beneficial for production industries like paper construction, polymer, and food processing. It is perceived that a single differential equation is described in the models of Newtonian fluid flows, but in the case of non-Newtonian fluid models, it is not so easy to describe the flow of the model with one and only constitutive differential equation. Usually, the rheological properties of fluids are specified with the help of their hypothetical constitutive conditions. Moreover, it is observed that the Newtonian fluids fulfilled 
Newton's internal friction law that is "shear stress is proportional to the viscosity of the fluid gradient" and nonNewtonian fluids dissatisfy the Newtonian law of internal friction. The leading flow equations of non-Newtonian fluids are more difficult than the Navier-Stokes equations [1-3]. Generally, non-Newtonian fluids are categorized into three dissimilar categories, namely, first is differential type, second is the integral type, and the third one is rate type. In the current research work, we observed the model of Jeffrey fluid flow, and this sort of fluid flow model indicates the property of the ratio of relaxation and retardation time. It is verified that the non-Newtonian models of fluid flows, with as well as without the magnetic-hydrodynamic field, have countless uses among the different fields of life, for example, biological fluids management, dental amalgam, plasmas, alloys, and metals that are in liquid form, electromagnetic propulsion, and blood $[4,5]$.

It is observed that the Jeffrey fluid is an extraordinary type of non-Newtonian fluid. Among many other types of nonNewtonian fluid models, it is revealed that the model of Jeffrey fluid flow is one of the substantial models which clearly precise the finest description of properties of the viscoelastic fluids [6-11]. It is presented that, in nature, Jeffrey fluid models are well-defined in linear viscoelastic fluids. As we are familiar with this, Jeffrey fluids have several types of applications in polymer industries and one of them is dilute polymer which is explained by the researchers Farooq et al. and Ara et al. $[12,13]$. Due to the viscoelastic behavior of Jeffrey fluid models, the vital and applicable role of such models is found in fluid mechanics and biological aspects. It has been magnificently used in the model of blood flow. As a special case, Jeffrey fluid is interconnected with Maxwell fluid and Newtonian fluid [14].

It is originated in physiology that the magnetohydrodynamic fields have countless applications, for example, magnetic devices and magnetic particles are used in the form of drug transferors, magnetic resonance imaging, and blood (biomagnetic fluid) is generated in the occurrence of hemoglobin molecules [15]. In 2011, Tripathi et al. observed the Jeffrey fluid peristaltic flow over a cylindrical tube that has finite length. They observe that fluid is electrically conducted in the occurrence of an applied magnetic field. The investigation takes place in the hypothesis of calculations of long wavelength and low Reynolds number [16]. Das [17] observed the behavior of different parameters during the study of the peristaltic flow of an electrically conducting incompressible viscous fluid through an inclined plan with the asymmetric channel. Raju C.S.K. et al. studied the impact of thermal radiation and chemical reaction on the boundary layer of a magnetohydrodynamic Jeffrey nanofluid flow above a permeable cone in the existence of Brownian motion and thermophoresis effects [18]. The mutual result of mass and heat transfer in Jeffrey fluid flow by a porous medium through a stretching sheet bound by transverse magnetic field in the existence of heat source/sink has been considered by Jena et al. [19]. Ellahi et al. [20] academically observe the model of Jeffrey fluid peristaltic flow through a rectangular duct which is nonuniform under the effects of ion and Hall slip. The obtained results for the Jeffrey fluid peristaltic flow disclose many remarkable behaviors that permit advanced research regarding non-Newtonian fluids, exclusively the shear-thinning phenomena. The influence of silver nanoparticles (AgNPs) and copper nanoparticles (CuNPs) on the MHD unsteady free convection Jeffrey fluid flow above a vertical oscillating plate fixed with a porous medium which is saturated is studied by Zin et al. [21], and they perceived that an increment in volume fraction causes the boost of temperature distribution and velocity profile. In contrast, the Hartmann number decreases the flow of the fluid due to the effect of Lorentz forces. Hayat et al. [22-24] studied the flow of MHD Jeffrey fluid under different circumstances and observed the behavior of velocity under the effect of embedded parameters. Sharma and Gupta [25] investigated in their paper the steady 2D flow of an incompressible MHD boundary layer flow and heat transfer of nanofluid above an impermeable surface in the occurrence of viscous dissipation and thermal radiation. Sana et al. investigate the Jeffrey flow over a permeable wall [26]. Furthermore, Jeffrey fluid flow, MHD and porosity, porous medium, and permeable wall have been discussed by various authors in [27-41].

In this research work, we study the MHD Jeffrey fluid model along with the porosity parameter because it is verified that, for practical determination, it is very beneficial in sciences and engineering fields. It is presented in countless fields such as electrochemistry finance, electromagnetism, biochemistry, and signal process, and the electrical conducting fluid flow is the vital application of magnetic field; MHD effect is used to deal with the dynamics of fluids which are electrically conducted. Furthermore, the MHD stream problem has amplified a noteworthy role in the aspects of its wide-ranging applications in medical and engineering fields.

The principles of MHD are applied in the strategy, design and scheme of pumps, heat exchangers, radar systems, power generators, flow meters, and many more. In this work, we consider the MHD effect with porous medium not rigid.

The main objective of this research work is to observe the behavior of velocity of Jeffrey fluid's transient flow under the influence of different embedded parameters along with MHD and porosity effects. For this purpose, Jeffrey fluid flow is observed over an infinite accelerated plate which is taken to be porous. The governing equations with initial and boundary conditions of the model are transformed to a nondimensional form by using an appropriate set of dimensionless variables. With the help of Laplace Transformation and perturbation methods, we attain analytical solutions for the velocity distribution of the Jeffrey fluid. In the end, to check the effect of different parameters on velocity we plot graphs by using Mathcad software, and it is observed how velocity distribution increases or decreases by increasing or decreasing the values of these parameters.

\section{Governing Equations of Jeffrey Fluid Model}

We take the velocity field for the current problem as [40]

$$
V=\left(u(y, t), V_{0}, 0\right),
$$


where $u(y, t)$ is the velocity distribution in $x$-direction and $V_{0}$ is the velocity of fluid in $y$-direction.

The constitutive equations for a Jeffrey fluid are [41]

$$
\begin{aligned}
& \tau=-p \mathrm{I}+\mathrm{S} \\
& \mathrm{S}=\frac{\mu}{1+\lambda_{1}}\left[\mathrm{R}_{1}+\lambda_{2}\left(\frac{\partial \mathrm{R}_{1}}{\partial t}+V . \nabla\right) \mathrm{R}_{1}\right]
\end{aligned}
$$

where $\tau$ implies Cauchy stress tensor, $\mathrm{S}$ represents extra stress tensor, $\mu$ is the dynamic viscosity of the fluid, $\lambda_{1}$ is the ratio of relaxation and retardation times, $\lambda_{2}$ is the retardation time, and $R_{1}$ is the Rivlin-Ericksen tensor which is welldefined as follows:

$$
\mathrm{R}_{1}=(\nabla V)+(\nabla V)^{t} .
$$

\section{Mathematical Modeling}

Let us examine the incompressible transient flow of the MHD Jeffrey fluid model covering the space lying above an accelerating plate, the plate is taken to be porous. It is presented that the velocity field of the fluid is consider of the form (1).

A uniform transverse magnetic field of strength $B_{0}$ is applied parallel to the $y$-axis. In beginning, at $t=0$, the velocity profile of Jeffrey fluid flow will be zero. At time $t=$ $0^{+}$, the velocity of the porous plate is $U_{0} e^{i \omega t}$. Figure 1 is shown as follows.

In the light of above observations for the given Jeffrey fluid model, the governing equation for the flow of unsteady incompressible MHD Jeffrey fluid over a porous plate has the resulting form $[26,29]$ :

$$
\begin{aligned}
\rho\left(\frac{\partial u}{\partial t}-V_{0} \frac{\partial u}{\partial y}\right)= & \frac{\mu}{1+\lambda_{1}}\left[\frac{\partial^{2} u}{\partial y^{2}}+\lambda_{2} \frac{\partial^{3} u}{\partial y^{2} \partial t}+\lambda_{2} V_{0} \frac{\partial^{3} u}{\partial y^{3}}\right] \\
& -\frac{\mu \phi}{\kappa\left(1+\lambda_{1}\right)}\left(1+\lambda_{2} \frac{\partial}{\partial t}\right) u-\sigma B_{0}^{2} u, y>0, t>0,
\end{aligned}
$$

where $u=u(y, t), v(y, t)=V_{0}$ are the components of velocity in the $x$ - and $y$-direction, respectively. $V_{0}$ indicates the uniform transpiration (blowing or suction) velocity at the surface of the permeable wall. $\lambda_{1}$ is the ratio of relaxation to retardation times, and $\lambda_{2}$ is the retardation time. It is notable that, by placing $\lambda_{1}=\lambda_{2}=0$, in the above listed equation, we attain the governing equation of Newtonian fluid, and if $\lambda_{1}=0, \lambda_{2} \neq 0$, then it is second grade fluid. $\mu$ shows the coefficient of fluid viscosity, $\rho$ represents the density of the fluid, $\kappa$ and $\phi$ denote the permeability and porosity of the porous plate, respectively, $\sigma$ is the fluid's electrical conductivity, and $B_{0}$ is the magnitude of applied magnetic field.

The appropriate initial and boundary conditions are [26]

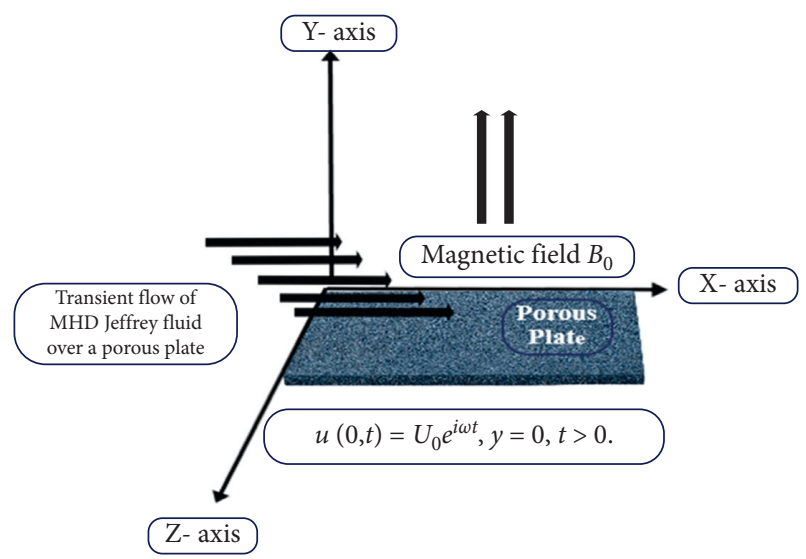

Figure 1: MHD Jeffrey fluid flow above an accelerated porous plate.

$$
\begin{aligned}
u(y, 0) & =0, \quad y>0, \\
u(0, t) & =u_{0}(t)=U_{0} e^{\imath \omega t} \quad \text { at } y=0, t>0, \\
u(\infty, t) & =0, \quad \text { as } y \longrightarrow \infty, t>0,
\end{aligned}
$$

where $\omega$ is the oscillating frequency.

Now, to obtain the dimensionless form of the above mention equations, we use the following set of dimensionless variables as [39]

$$
\begin{aligned}
u^{*} & =\frac{u}{U_{0}}, \\
y^{*} & =\frac{U_{0} y}{\nu}, \\
t^{*} & =\frac{U_{0}^{2} t}{\nu}, \\
\omega^{*} & =\frac{\omega \nu}{U_{0}^{2}}, \\
\beta & =\frac{U_{0}^{2}}{v} \lambda_{2}, \\
2 \gamma & =\frac{V_{0}}{U_{0}}, \\
\frac{1}{k} & =\frac{\nu^{2} \phi}{\kappa U_{0}^{2}}, \\
H a & =\frac{\sigma B_{0}^{2} v}{\rho U_{0}^{2}} .
\end{aligned}
$$

The nondimensional form of the initial-boundary value problem (for simplicity, dropping $\left({ }^{*}\right)$ notation) as 


$$
\begin{gathered}
\frac{1}{1+\lambda_{1}}\left(\frac{\partial^{2} u}{\partial y^{2}}+\beta \frac{\partial^{3} u}{\partial y^{2} \partial t}+2 \beta \gamma \frac{\partial^{3} u}{\partial y^{3}}\right) \\
-\frac{1}{k\left(1+\lambda_{1}\right)}\left(1+\beta \frac{\partial}{\partial t}\right) u-\text { Hau } \\
+2 \gamma \frac{\partial u}{\partial y}-\frac{\partial u}{\partial t}=0, \\
u(y, 0)=0, \quad y>0, \\
u(0, t)=u_{0}(t)=e^{i \omega \mathrm{t}} \quad \text { at } y=0, t>0, \\
u(\infty, t)=0, \quad \text { as } \mathrm{y} \longrightarrow \infty, t>0,
\end{gathered}
$$

where $\beta$ shows Jaffrey fluid parameter and $\gamma$ is the wall transpiration parameter $(\gamma>0$ for suction and $\gamma<0$ for injection). $k$ and $H a$ are the porosity and magnetic parameters.

\section{Investigate the Solution of the Velocity Field}

With the usage of the Laplace transformation method and a regular perturbation method, we get the solution of nondimensional initial boundary value problem (7)-(10). By applying the Laplace transformation method with respect to $t$ (time) on the above equations, we obtain the resulting equations as

$$
\begin{aligned}
& \frac{1}{1+\lambda_{1}}(1+\beta s) \frac{\partial^{2} \bar{u}}{\partial y^{2}}+\frac{2 \beta \gamma}{1+\lambda_{1}} \frac{\partial^{3} \bar{u}}{\partial y^{3}}+2 \gamma \frac{\partial \bar{u}}{\partial y}-s \bar{u} \\
& -\frac{1}{k\left(1+\lambda_{1}\right)}(1+\beta s) \bar{u}-H a \bar{u}=0, \\
& \bar{u}(0, s)=\frac{1}{s-\iota \omega}, \bar{u}(\infty, s) \\
& =0 a s y \longrightarrow \infty, t>0,
\end{aligned}
$$

where $\bar{u}(y, s)$ is named as preimage of $u(y, t)$ and $s$ is identified as transform parameter.

To find the solution of (11) by employing boundary conditions (12), we use regular perturbation technique and expand $\bar{u}(y, s)$ in terms of the parameter $\beta(\beta \ll 1)$ :

$$
\bar{u}(y, s)=\bar{u}_{0}(y, s)+\beta \bar{u}_{1}(y, s)+o\left(\beta^{2}\right) .
$$

Now, by substituting (13) into (11) and (12), and then equating the powers of $\beta$, we have

$$
\begin{aligned}
& \bar{u}_{0}^{\prime \prime}+2 \gamma\left(1+\lambda_{1}\right) \bar{u}_{0}^{\prime}-\left[\left(1+\lambda_{1}\right) s+\frac{1}{k}+H a\left(1+\lambda_{1}\right)\right] \bar{u}_{0}=0 \\
& \bar{u}_{0}(0, s)=\frac{1}{s-\iota \omega}, \\
& \bar{u}_{0}(\infty, s)=0 \\
& \bar{u}_{1}^{\prime \prime}+2 \gamma\left(1+\lambda_{1}\right) \bar{u}_{1}^{\prime}-\left[\left(1+\lambda_{1}\right) s+\frac{1}{k}+H a\left(1+\lambda_{1}\right)\right] \bar{u}_{1} \\
& =-2 \gamma \bar{u}_{0}^{\prime \prime}-s \bar{u}_{0}^{\prime \prime}+\frac{s}{k} \bar{u}_{0}, \\
& \bar{u}_{1}(0, s)=0
\end{aligned}
$$$$
\bar{u}_{1}(\infty, s)=0 .
$$

The solutions of the above equations are as follows:

$$
\begin{aligned}
& \bar{u}_{0}(y, s)=\frac{1}{s-\iota \omega} e^{-y\left(\delta_{0}+\delta_{1} \sqrt{s+\delta_{2}}\right)}, \\
& \bar{u}_{1}(y, s)=-\frac{D_{1}(s) y}{2 \delta_{1} \sqrt{s+\delta_{2}}} e^{-y\left(\delta_{0}+\delta_{1} \sqrt{s+\delta_{2}}\right)},
\end{aligned}
$$

where

$$
\begin{aligned}
\delta_{0}= & \gamma\left(1+\lambda_{1}\right), \\
\delta_{1}= & \sqrt{1+\lambda_{1}}, \\
\delta_{2}= & \frac{\gamma^{2}\left(1+\lambda_{1}\right)^{2}+1 / k+H a\left(1+\lambda_{1}\right)}{\left(1+\lambda_{1}\right)}, \\
D_{1}(s)= & \frac{2 \gamma\left(\delta_{0}+\delta_{1} \sqrt{s+\delta_{2}}\right)^{3}}{s-\iota \omega}-\frac{s\left(\delta_{0}+\delta_{1} \sqrt{s+\delta_{2}}\right)^{2}}{s-\iota \omega} \\
& +\frac{s}{k(s-\iota \omega)} .
\end{aligned}
$$

Perturbation solution of the given problem is

$$
\bar{u}(y, s)=\left[\frac{1}{s-\iota \omega}-\beta \frac{D_{1}(s) y}{2 \delta_{1} \sqrt{s+\delta_{2}}}\right] e^{-y\left(\delta_{0}+\delta_{1} \sqrt{s+\delta_{2}}\right)} .
$$

Applying inverse Laplace transform to (15) and (16), 


$$
\begin{aligned}
u_{0}(y, t)= & e^{-\delta_{0} y-\delta_{2} t} \operatorname{erfc}\left(\frac{\delta_{1} y}{2 \sqrt{t}}\right)+\left(\delta_{2}+\iota \omega\right) e^{-\delta_{0} y} \\
& \int_{0}^{t} e^{\iota \omega(t-\tau)} e^{-\delta_{2} \tau} \operatorname{erfc}\left(\frac{\delta_{1} y}{2 \sqrt{\tau}}\right) d \tau \\
u_{1}(y, t)= & -\frac{y}{2 \delta_{1}} d_{1}(t) \cdot d_{2}(2) .
\end{aligned}
$$

Now, from (19) and (20), we obtain velocity field as

$$
\begin{aligned}
u(y, t)= & e^{-\delta_{0} y-\delta_{2} t} \operatorname{erfc}\left(\frac{\delta_{1} y}{2 \sqrt{t}}\right) \\
& +\left(\delta_{2}+\iota \omega\right) e^{-\delta_{0} y} \int_{0}^{t} e^{\iota \omega(t-\tau)} e^{-\delta_{2} \tau} \operatorname{erfc}\left(\frac{\delta_{1} y}{2 \sqrt{\tau}}\right) d \tau \\
& -\beta \frac{y}{2 \delta_{1}} d_{1}(t) d_{2}(t)
\end{aligned}
$$

where

$$
\begin{aligned}
& d_{1}(t)=e_{1}(t) \cdot e_{2}(t)+e_{3}(t), \\
& d_{2}(t)=y \delta_{1} \frac{e^{-y^{2} \delta_{1}^{2} / 4 t}}{2 t \sqrt{\pi t}} \cdot e^{-y \delta_{0}-\delta_{2} t}, \\
& e_{1}(t)=\frac{\delta_{0}^{2} e^{-\delta_{2} t}}{\sqrt{\pi t}}-\delta_{1}^{2} \frac{e^{-\delta_{2} t}}{2 t \sqrt{\pi t}}+2 \delta_{0} \delta_{1} \delta(t), \\
& e_{2}(t)=-\delta(t)+\left(2 \gamma \delta_{0}-\iota \omega\right) e^{\imath \omega t}+2 \gamma \delta_{1} e^{\imath \omega t}\left(-\frac{e^{-\delta_{2} t}}{2 t \sqrt{\imath t}}\right), \\
& e_{3}(t)=\frac{e^{-\delta_{2} t}}{k \sqrt{\pi t}}+\frac{\iota \omega}{k} e^{\imath \omega t}\left(\frac{e^{-\delta_{2} t}}{\sqrt{\pi t}}\right) .
\end{aligned}
$$

Here, $\delta(t)$ is a Dirac delta function.

4.1. Limiting Case. The following figure shows a comparison of the present work in the absence and presence of MHD and porosity effects. It is clear from this figure that, in the presence of MHD and porosity effects, the velocity decays early when compared with the present solutions in the absence of MHD and porosity effects (Figure 2).

\section{Discussion of Numerical Results and Graphs}

With the purpose of arguing certain physical characteristics of the calculated solution, graphs have been prepared for the velocity profile of the fluid.

The influence of time is important to explain here. Figure 3 reveals the behavior of velocity field of fluid with altered values of $t$, and it is prominent from this diagram that the velocity field boosts by increasing the values of time $t$. Figure 4 is drawn to observe the flow of velocity field under the impact of parameter, the ratio of relaxation and retardation time, $\lambda_{1}$. It is revealed that the velocity profile is

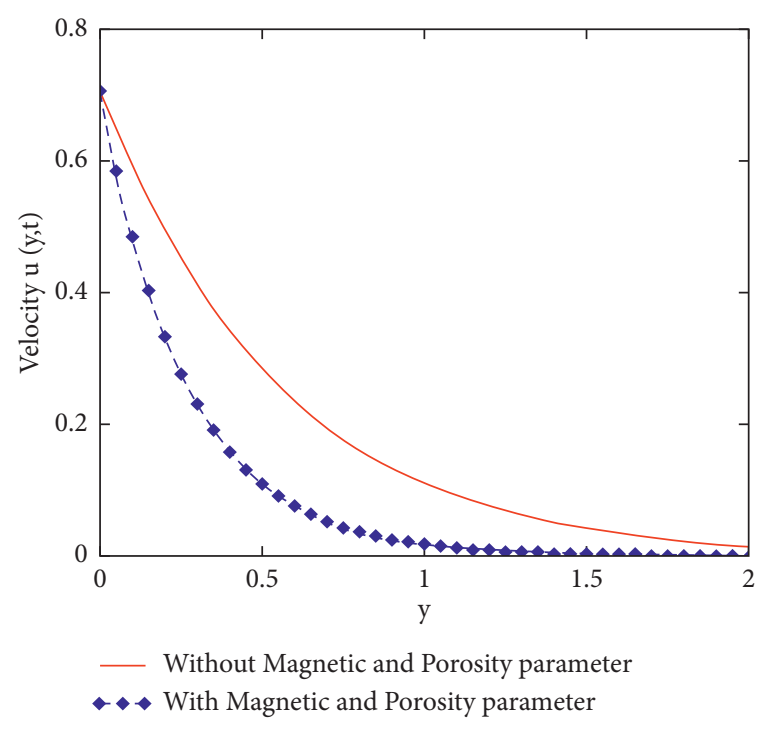

FIGURE 2: Comparison of velocity field with and without magnetic and porosity parameters.

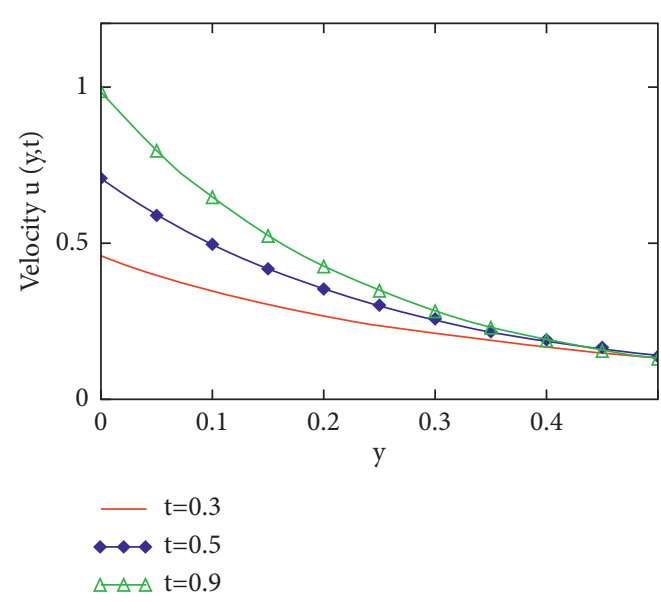

Figure 3: Velocity profile for different values of time ( $t$ ).

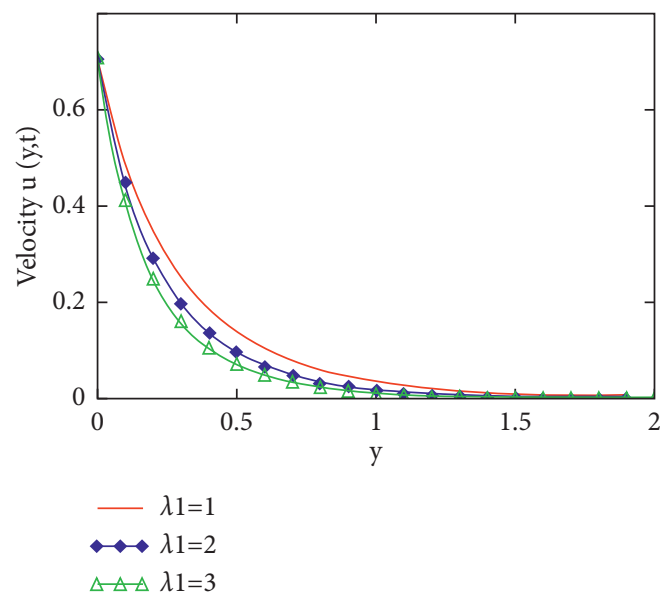

FIGURE 4: Velocity profile for different values of parameter ratio of relation and retardation time $\lambda_{1}$. 


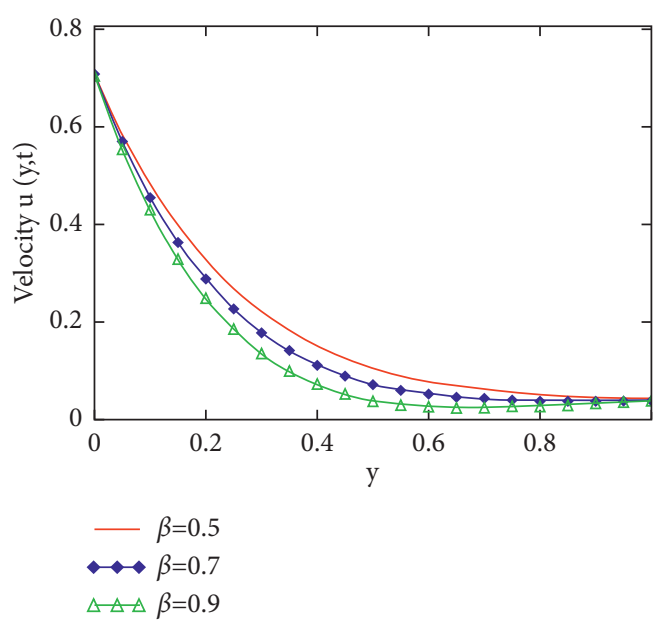

Figure 5: Velocity profile for different values of Jeffrey fluid parameter $\beta$.

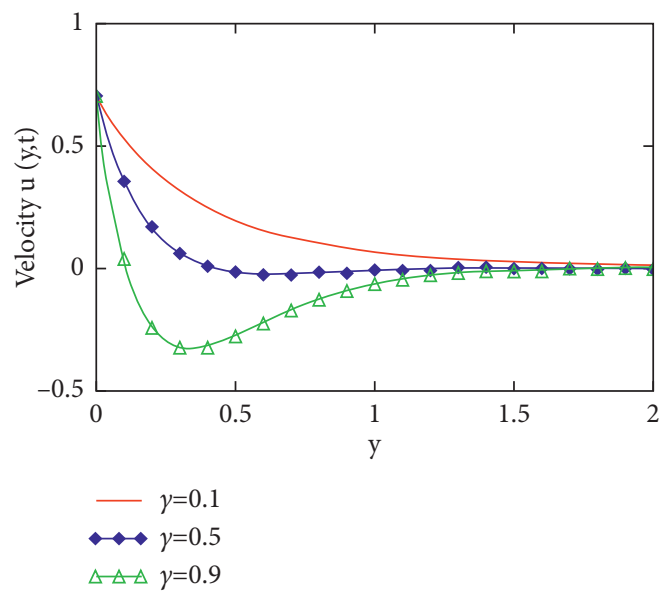

FIGURE 6: Velocity profile for different values of transpiration parameter $\gamma$.

declined with increased parameter $\lambda_{1}$ because higher values of $\lambda_{1}$ lead to growth in relaxation time (or decay in retardation time), it means that the particles of the fluid require much more time to come back in equilibrium system from a perturbed system. Consequently, velocity of the fluid decreases. The variation in velocity distribution of the fluid by changing the values of Jeffrey fluid parameter $\beta$ is observed in Figure 5. It is perceived that the velocity profile declines as the values of Jeffrey fluid parameter increases. The reason behind the decrease in velocity profile is that, as we increase the values of Jaffrey fluid parameter, the boundary layer momentum thickness will rise. Hence, the velocity distribution declines as the values of $\beta$ rises up. By observing Figure 6, we notice the behavior of velocity distribution under the effect of wall transpiration parameter $\gamma$. It is noticed that the velocity of Jeffrey fluid flow decreases along with increasing wall transpiration (injection/suction parameter) parameter. In actual fact, with the porous boundaries, the fluid experiences greater viscosity and therefore offers resistance to flow, and this leads to reduce in

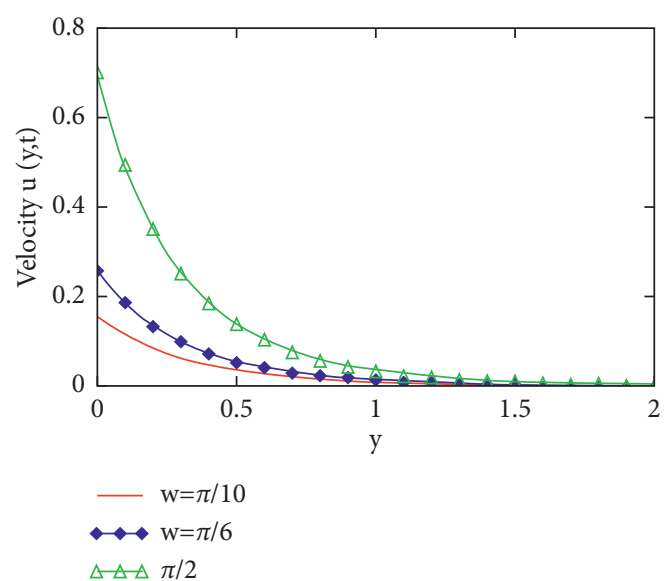

Figure 7: Velocity profile for different values of oscillating frequency $\omega$.

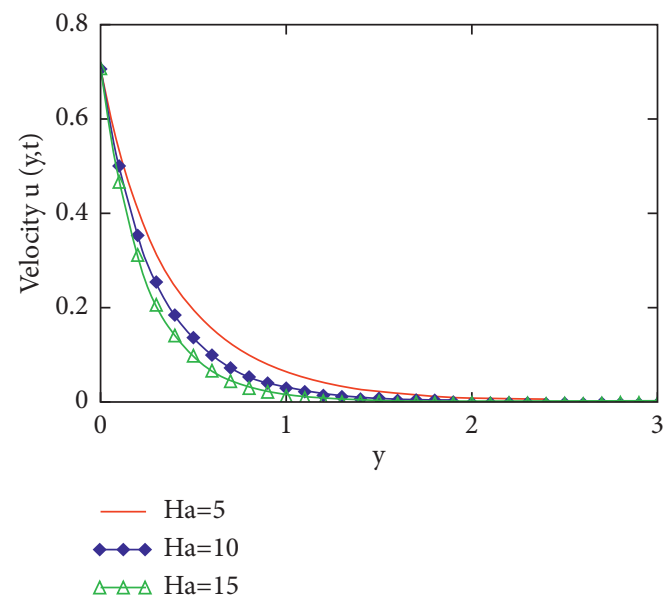

FIGURE 8: Velocity profile for different values of magnetic parameter $\mathrm{Ha}$.

the velocity. For the large values of parameter, the decay is more. With the help of Figure 7, we examine the influence of oscillation frequency $\omega$. It is noticed that the Jeffrey fluid's velocity is a strong function of $\omega$, and its effect on the velocity profile is opposite as that of the ratio of relaxation and retardation time $\lambda_{1}$. With the help of Figure 8, we understand that how velocity distribution reacts against magnetic parameter $H a$. We perceived that velocity profile diminishes upon increasing the values of magnetic parameter. It happens because of the Lorentz force, which appears when a magnetic field imposes to an electrically conducting fluid and a drag force is produced. Because of this force, fluid motion slows down near the plate and away from the plate; all other forces including Lorentz force weaken as a result when the fluid comes to rest. The behavior of porosity parameter $\kappa$ is demonstrated by Figure 9. It is realized that the velocity field accelerates due to the porosity parameter $\kappa$. We observed that, with the growth in permeability of porous medium, the drag force decreases; for this reason, velocity profile of the fluid accelerates. Figure 10 plots suctions and 


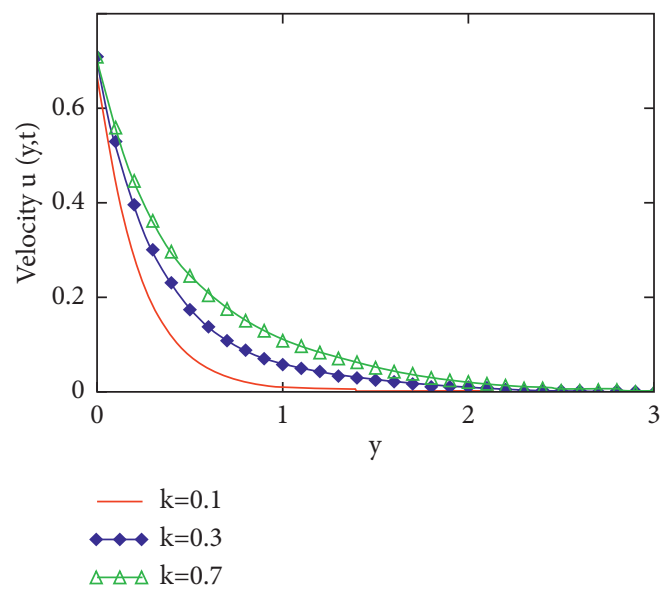

FIGURE 9: Velocity profile for different values of porosity parameter $\kappa$

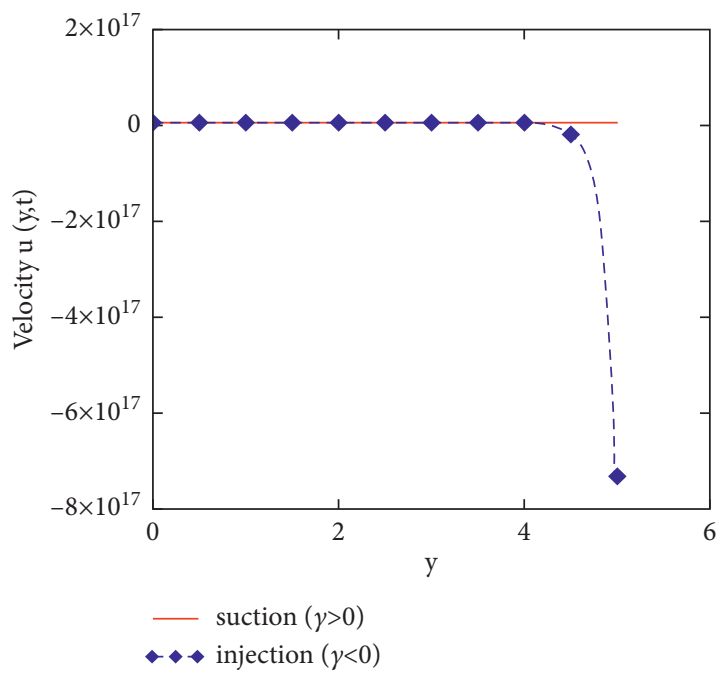

Figure 10: Velocity profile for suction and injection.

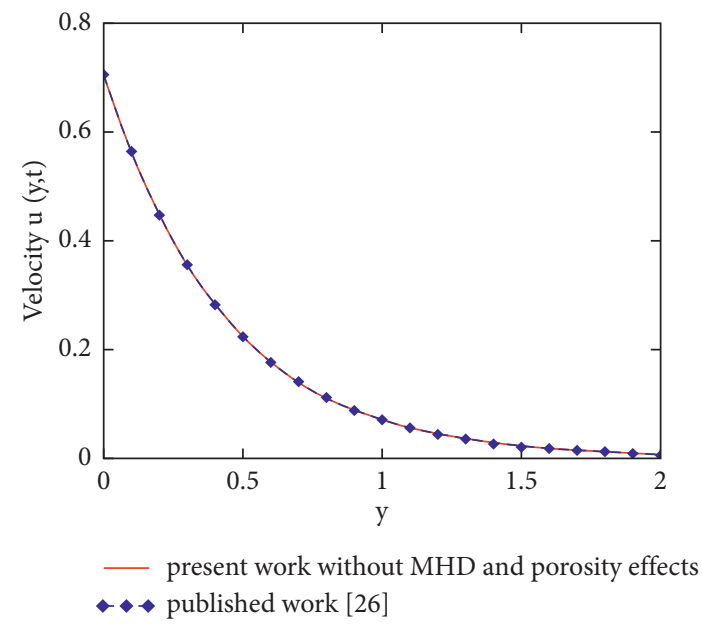

Figure 11: Comparison with published work [26]. injection velocities, whereas Figure 11 shows the comparison of the present work with the published work, and an excellent agreement is found.

\section{Conclusion}

This research work presents approximate analytical solutions for the unsteady flows of Jeffrey fluids over a porous, oscillating plate with MHD and porosity effect.

Approximate analytical solutions for the nondimensional velocity field in the transformed domain have been obtained using the Laplace transform and perturbation method.

Graphs have been plotted to examine the effect of embedded parameters on the velocity profile of the fluid. The following observation is perceived by these graphs:

(i) The velocity distribution increases with the increasing values of the time.

(ii) By increasing the parameter ratio of relaxation and retardation time, it leads to decay in retardation time in result the velocity profile is decreasing.

(iii) As the values of Jaffrey fluid parameter increased, the velocity distribution is declined due to the rise in boundary layer momentum thickness.

(iv) The velocity of Jeffrey fluid flow is decreased upon increasing the wall transpiration parameter because the fluid experience greater viscosity and resist to flow.

(v) Effect of oscillation frequency parameter on velocity profile is opposite as that of parameter ratio of relaxation and retardation time.

(vi) Velocity profile diminishes upon increasing magnetic parameter because of Lorentz forces which appear under the MHD effect.

(vii) By increasing the values of porosity parameter, drag forces decreases; hence, the velocity distribution also increased.

(viii) In future, this work will be extended for other nonNewtonian fluids, nanofluids or by changing the physical frame work. We will also consider skin friction and temperature field in our extended work.

\section{Nomenclature}

$u(y, t)$ : Velocity distribution in $x$-direction

$V_{0}$ : Velocity distribution in $y$-direction

$\mu: \quad$ Dynamic viscosity

$\rho: \quad$ Constant fluid's density

$\lambda_{1}$ : $\quad$ Ratio of relaxation time to the retardation time

$\lambda_{2}: \quad$ Retardation time

$\beta$ : $\quad$ Jeffrey fluid parameter

$\gamma: \quad$ Wall transpiration

$\omega: \quad$ Oscillations frequency

$s$ : $\quad$ Laplace transform parameter

$\delta(t): \quad$ Dirac distribution 
$\tau$ : $\quad$ Cauchy stress tensor

S: $\quad$ Extrastress tensor

p: $\quad$ Pressure of the fluid

I: $\quad$ Identity tensor

$R_{1}$ : Rivlin-Ericksen tensor

$\nabla V: \quad$ Gradient of the vector field $V$

$V \nabla: \quad$ Divergence of vector field $V$

$t: \quad$ Time

$B_{0}: \quad$ Magnetic field strength

$\kappa: \quad$ Permeability of the medium

$\phi: \quad$ Porosity parameter

$k=\phi / \kappa:$ Porosity constant

$\sigma: \quad$ Electrical conductivity

$v$ : Kinematic viscosity

Ha: Hartmann number (magnetic parameter).

MHD: Magneto hydrodynamic.

\section{Data Availability}

No data were used to support this study.

\section{Conflicts of Interest}

The authors declare that they have no conflicts of interest.

\section{References}

[1] A. Khan, G. Zaman, S. Ahmad, and M. I. Chohan, "Some exact solutions of generalized Jeffrey fluid using N-transform," International Journal of Computational Mathematics, vol. 7, no. 4, p. 402, 2017.

[2] S. Han, L. Zheng, and X. Zhang, "Slip effects on a generalized Burgers' fluid flow between two side walls with fractional derivative," Journal of the Egyptian Mathematical Society, vol. 24, no. 1, pp. 130-137, 2016.

[3] M. Jamil, "Effects of slip on oscillating fractionalized Maxwell fluid," Nonlinear Engineering, vol. 5, no. 1, pp. 25-36, 2016.

[4] M. Khan, K. Maqbool, and T. Hayat, "Influence of Hall current on the flows of a generalized Oldroyd-B fluid in a porous space," Acta Mechanica, vol. 184, no. 1-4, pp. 1-3, 2006.

[5] T. Hayat, M. Waqas, S. A. Shehzad, and A. Alsaedi, "MHD stagnation point flow of Jeffrey fluid by a radially stretching surface with viscous dissipation and Joule heating," Journal of Hydrology and Hydromechanics, vol. 63, no. 4, pp. 311-317, 2015.

[6] M. Khan, "Partial slip effects on the oscillatory flows of a fractional Jeffrey fluid in a porous medium," Journal of Porous Media, vol. 10, no. 5, pp. 473-488, 2007.

[7] S. V. Jakati, B. T. Raju, A. L. Nargund, and S. B. Sathyanarayana, "The effect of Brownian motion and thermophoresis on nanofluids stretching for Jeffrey fluid model," International Journal of Latest Transactions in Engineering and Science, vol. 3, no. 3, pp. 1-9, 2018.

[8] S. A. Shehzad, T. Hayat, M. S. Alhuthali, and S. Asghar, "MHD three-dimensional flow of Jeffrey fluid with Newtonian heating," Journal of Central South University, vol. 21, no. 4, pp. 1428-1433, 2014.

[9] F. Sultan, N. A. Khan, M. Qasim, and M. I. Afridi, "Numerical simulation of the flow of nano-Eyring-Powell fluid through a curved artery with time-variant stenosis and aneurysm," Nihon Reoroji Gakkaishi, vol. 47, no. 2, pp. 75-85, 2019.
[10] K. Raju, A. Parandhama, M. C. Raju, and B. K. Ramesh, "Unsteady MHD free convection Jeffery fluid flow of radiating and reacting past a vertical porous plate in slip-flow regime with heat source," Frontiers in Heat and Mass Transfer, vol. 10, 2018.

[11] S. Nadeem, A. Hussain, and K. Majid, "Stagnation flow of a Jeffrey fluid over a shrinking sheet," Zeitschrift für Naturforschung A, vol. 65, no. 6-7, pp. 540-548, 2010.

[12] M. Farooq, N. Gull, A. Alsaedi, and T. Hayat, "MHD flow of a Jeffrey fluid with Newtonian heating," Journal of Mechanics, vol. 31, no. 3, pp. 319-329, 2015.

[13] A. Ara, N. A. Khan, F. Sultan, and S. Ullah, "Numerical simulation of Jeffery-Hamel flow Bingham plastic fluid and heat transfer in the presence of magnetic field," Applied and Computational Mathematics, vol. 18, no. 2, pp. 135-148, 2019.

[14] A. S. Rao, N. Nagendra, and V. R. Prasad, "Heat transfer in a non-Newtonian jeffrey's fluid over a non-isothermal wedge," Procedia Engineering, vol. 127, pp. 775-782, 2015.

[15] T. Hayat, M. Rafiq, and B. Ahmad, "Soret and Dufour effects on MHD peristaltic flow of Jeffrey fluid in a rotating system with porous medium," PloS One, vol. 11, no. 1, p. e0145525, 2016.

[16] D. Tripathi, N. Ali, T. Hayat, M. K. Chaube, and A. A. Hendi, "Peristaltic flow of MHD Jeffrey fluid through finite length cylindrical tube," Applied Mathematics and Mechanics, vol. 32, no. 10, pp. 1231-1244, 2011.

[17] K. Das, "Influence of slip and heat transfer on MHD peristaltic flow of a Jeffrey fluid in an inclined asymmetric porous channel," Indian Journal of Mathematics, vol. 54, no. 1, pp. 19-45, 2012.

[18] C. S. Raju, M. Jayachandra Babu, and N. Sandeep, "Chemically reacting radiative MHD Jeffrey nanofluid flow over a cone in porous medium," International Journal of Engineering Research in Africa, vol. 19, pp. 75-90, 2016.

[19] S. Jena, S. R. Mishra, and G. C. Dash, "Chemical reaction effect on MHD Jeffery fluid flow over a stretching sheet through porous media with heat generation/absorption," International Journal of Algorithms, Computing and Mathematics, vol. 3, no. 2, pp. 1225-1238, 2017.

[20] R. Ellahi, M. M. Bhatti, and I. Pop, "Effects of hall and ion slip on MHD peristaltic flow of Jeffrey fluid in a non-uniform rectangular duct," International Journal of Numerical Methods for Heat and Fluid Flow, vol. 26, no. 6, pp. 1802-1820, 2016.

[21] N. A. Zin, I. Khan, and S. Shafie, "The impact silver nanoparticles on MHD free convection flow of Jeffrey fluid over an oscillating vertical plate embedded in a porous medium," Journal of Molecular Liquids, vol. 222, pp. 138-150, 2016.

[22] T. Hayat, R. Sajjad, and S. Asghar, "Series solution for MHD channel flow of a Jeffery fluid," Communications in Nonlinear Science and Numerical Simulation, vol. 15, no. 9, pp. 24002406, 2010.

[23] T. Hayat, G. Bashir, M. Waqas, and A. Alsaedi, "MHD flow of Jeffrey liquid due to a nonlinear radially stretched sheet in presence of Newtonian heating," Results in Physics, vol. 6, pp. 817-823, 2016.

[24] T. Hayat, S. Qayyum, M. Imtiaz, and A. Alsaedi, "Three-dimensional rotating flow of Jeffrey fluid for Cattaneo-Christov heat flux model," AIP Advances, vol. 6, no. 2, Article ID 025012, 2016.

[25] K. Sharma and S. Gupta, "Viscous dissipation and thermal radiation effects in MHD flow of Jeffrey nanofluid through impermeable surface with heat generation/absorption," Nonlinear Engineering, vol. 6, no. 2, pp. 153-166, 2017. 
[26] S. Bajwa, S. Ullah, I. Khan, and M. Fayz-Al-Asad, "Transient flow of Jeffrey fluid over a permeable wall," Mathematical Problems in Engineering, vol. 2021, Article ID 9999949, 9 pages, 2021.

[27] D. Yang, M. Israr Ur Rehman, A. Hamid, and S. Ullah, "Multiple solutions for stagnation-point flow of unsteady carreau fluid along a permeable stretching/shrinking sheet with non-uniform heat generation," Coatings, vol. 11, no. 9, p. 1012, 2021.

[28] N. A. Khan, F. Naz, N. A. Khan, and S. Ullah, "MHD nonaligned stagnation point flow of second grade fluid towards a porous rotating disk," Nonlinear Engineering, vol. 8, no. 1, pp. 231-249, 2019.

[29] N. A. Khan, S. Khan, and S. Ullah, "MHD flow of burger's fluid over an off-centred rotating disk in a porous medium," AIP Advances, vol. 5, no. 8, pp. 1-25, 2015.

[30] N. Acharya, S. Maity, and P. K. Kundu, "Differential transformed approach of unsteady chemically reactive nanofluid flow over a bidirectional stretched surface in presence of magnetic field," Heat transfer, vol. 49, no. 6, pp. 3917-3942, 2020.

[31] N. Acharya, H. Mondal, and P. K. Kundu, "Spectral approach to study the entropy generation of radiative mixed convective couple stress fluid flow over a permeable stretching cylinder," Proceedings of the Institution of Mechanical Engineers - Part C: Journal of Mechanical Engineering Science, vol. 235, no. 15, pp. 2692-2704, 2021.

[32] N. Acharya, K. Das, and P. K. Kundu, "Influence of multiple slips and chemical reaction on radiative MHD Williamson nanofluid flow in porous medium: a computational framework," Multidiscipline Modeling in Materials and Structures, vol. 15, 2019.

[33] V. K. Sinha, B. Kumar, G. S. Seth, and R. Nandkeolyar, "Features of Jeffrey fluid flow with Hall current: a spectral simulation," Pramana, vol. 94, no. 1, pp. 1-8, 2020.

[34] S. Nandi and B. Kumbhakar, "Hall current and thermo-diffusion effects on magnetohydrodynamic convective flow near an oscillatory plate with ramped type thermal and solutal boundary conditions," Indian Journal of Physics, pp. 1-14, 2021.

[35] S. Nandi and B. Kumbhakar, "Unsteady MHD free convective flow past a permeable vertical plate with periodic movement and slippage in the presence of Hall current and rotation," Thermal Science and Engineering Progress, vol. 19, Article ID 100561, 2020.

[36] C. Fetecau, D. Vieru, and A. Zeeshan, "Analytical solutions for two mixed initial-boundary value problems corresponding to unsteady motions of Maxwell fluids through a porous plate channel," Mathematical Problems in Engineering, vol. 2021, Article ID 5539007, 13 pages, 2021.

[37] A. Riaz, A. Zeeshan, M. M. Bhatti, and R. Ellahi, "Peristaltic propulsion of Jeffrey nano-liquid and heat transfer through a symmetrical duct with moving walls in a porous medium," Physica A: Statistical Mechanics and Its Applications, vol. 545, Article ID 123788, 2020.

[38] A. Zeeshan, N. Ijaz, A. Riaz, A. B. Mann, and A. Hobiny, "Flow of nonspherical nanoparticles in electromagnetohydrodynamics of nanofluids through a porous medium between eccentric cylinders," Journal of Porous Media, vol. 23, no. 12, 2020.

[39] K. Maqbool, A. B. Mann, and M. H. Tiwana, "Unsteady MHD convective flow of a Jeffery fluid embedded in a porous medium with ramped wall velocity and temperature," Alexandria Engineering Journal, vol. 57, no. 2, pp. 1071-1078, 2018.
[40] M. Jamil and A. Haleem, "MHD fractionalized Jeffrey fluid over an accelerated slipping porous plate," Nonlinear Engineering, vol. 9, no. 1, pp. 273-289, 2020.

[41] M. Qasim, "Heat and mass transfer in a Jeffrey fluid over a stretching sheet with heat source/sink," Alexandria Engineering Journal, vol. 52, no. 4, pp. 571-575, 2013. 